\title{
Interventional Oncology: A Key Part of Mainstream Cancer Care
}

Thank you very much indeed for that generous introduction. I am grateful to the organizing committee for including me in the excellent program for this meeting. I shall talk to you about interventional oncology this afternoon and why I consider it to be an important part of modern cancer care. However before that, I thought you might be interested in a few remarks on the names and locations of some London hospitals: I work at St. Thomas's, which is opposite Big Ben on the south bank of the Thames. However, was not always there: until the mid-1800s, it was a couple of miles away, in the London borough of Southwark, in St. Thomas's Street. However, the hospital currently of St. Thomas's street is Guy's Hospital. How is that? Well, Guy's was founded by people from St. Thomas's in 1725 . When that happened, St. Thomas's start considering moving away, and eventually got round to it about 100 years later. Hence, Guy's is in St. Thomas's street. I trained in Radiology at the Royal Postgraduate Medical School, at Hammersmith Hospital. When I was there, it was quite common for medical students from abroad doing elective periods at The Hammersmith to get on the underground station at Heathrow Airport, get out at Hammersmith station and-not unreasonably you might-think ask for the location of Hammersmith Hospital. Only to be told that, of course, Hammersmith Hospital was not in Hammersmith. Hammersmith hospital is in East Acton. There is a teaching hospital in Hammersmith: it is called Charing Cross Hospital. Moreover, there is no hospital in Charing. Cross, All very quirky I think.

There are three forms of local tumor treatment: surgery, radiotherapy and various interventional radiological methods, which collectively are called interventional oncology. Surgery will always be there because it's the only form of treatment that can deal with tumors in hollow organs, or indeed with large tumors anywhere. However, as we all know, tumors are getting smaller. Modern imaging can now reveal lesions down to about 3-4 $\mathrm{mm}$ in size, and I think it is inconceivable that 10 years from now tiny lesions such as this small metastasis is in the right lobe of the liver will be dealt with by removing a large segment of a solid organ. It is more likely that imaging-guided methods will be used. For practical purposes, this means either sophisticated new methods of radiation therapy or interventional radiological techniques. However, before we come to those, it is worth reminding ourselves that interventional radiologists have been contributing to the palliation of cancer for many years, using a variety of techniques. Just by way of a reminder or an illustration, let me show you the computed tomography (CT) of a patient who had surgery for esophageal cancer. The operation included a gastric pull-up, so the stomach was placed in the chest. After the operation, he developed a fistula between the stomach and the right main bronchus and his life was miserable because every time he had anything to eat or drink some of the food or drink went into the lungs, which is why you are looking at bilateral pneumonia. It would have been difficult to deal with this surgically in a frail patient, but it was straightforward to sort it out radiologically. We introduced a self-expanding stent covered with plastic, which sealed the fistula quite effectively. An esophagogram demonstrated flow in the remnant of the esophagus and into the small bowel without any of the contrast medium entering the lungs. This procedure transformed the patient's quality of life because he could now eat and drink again.

However, it is potentially curative methods of treatment, such as radioembolization, cryotherapy and thermal ablation that have put interventional oncology on the map. It all started with the thermal ablation in the liver and whenever I took about the liver I like to show this painting by Rubens, which shows Prometheus, who stole the fire from the gods, being punished for his impertinence by having his liver eaten by an eagle. I like this painting partly because of its exquisite quality, but also because of its anatomical accuracy. This eagle knows exactly where the liver is. It was not always so in older depictions of the same scene: on this Greek pot this eagle is about to give this Prometheus a pneumothorax. As for this Prometheus... he is getting really worried.

Radioembolization is a form of intravascular brachytherapy. It uses microspheres containing yttrium-90, which is a $\beta$-emitter, to destroy malignant cells at a range of about ten microns from the microsphere. In a phase 2 randomized study, 179 patients with hepatocellular carcinoma were divided between selective internal radiation therapy and transarterial chemoembolization on an intention to treat basis. Radioembolization was associated with significantly longer time to progression. It also works in patients with colorectal liver metastases: the Foxfire study compared radioembolization plus chemotherapy with chemotherapy alone, and found that there was no prolongation of overall survival in the totality of the group. However, in patients with right-sided primary tumors, which are biologically different, there was significantly longer overall survival and $36 \%$ reduction in the risk of death.

Radiofrequency ablation (RFA) is the mainstay of treatment in interventional oncology. This patient had RFA for a small colorectal liver metastasis. The CT showed a large area of coagulation after the procedure, with a reaction in the surrounding liver. A comparison of radiofrequency with hepatic resection in a meta-analysis in patients with small hepatocellular carcinomas found no difference in overall 
survival. RFA had a higher recurrence rate but hepatic resection had more complications.

In the iconic CLOCC study, 119 patients with colorectal liver metastases were followed up for almost 10 years. The median overall survival was longer with the combined treatment of RFA plus chemotherapy compared with chemotherapy alone. The 8-year overall survival with chemotherapy alone was $8.9 \%$, whereas with RFA plus chemotherapy it was $35.9 \%$. I think few people today would consider it reasonable to use chemotherapy alone in this group of patients.

Thermal ablation is an effective treatment for lung cancer. Here, we have an adenocarcinoma with activity on positron-emission tomography. The CT shows a spiculated lesion. Two years after treatment with RFA the activity has gone and the CT shows only a scar in the lung. The results are good. Cancer-specific survival for stage la nonsmall cell lung cancer was $52 \%$ at 5 years. The problem with RFA in the lung is the high local recurrence rate: in this study there was 33\% recurrence in the tumor bed. The good news is that, provided that you can treat the recurrent tumor, the high recurrence rate does not affect the rate of overall survival.

Microwave is newer and we do not yet have substantial studies comparing this form of treatment with RFA. The available evidence suggests that microwave produces larger areas of coagulation and probably has a lower recurrence rate than RFA. If this is confirmed in larger studies then it is likely that microwave will replace RFA in the lung.

Perhaps, the best application of thermal ablation is in the kidney. Here, we have a 42-mm renal cell carcinoma at the lower pole of the left kidney. A few months after treatment with RFA we have the area of coagulation separating from the normal parenchyma, with a reaction in the surrounding fat.

We have carried out a systematic review and meta-analysis comparing partial nephrectomy with thermal ablation for small renal tumors. We looked at a large number of studies and analyzed six in detail, comprising 587 patients with a mean tumor size of $2.5 \mathrm{~cm}$. There was no difference in local recurrence or disease-free survival. Complications were more common with surgery. The most significant finding was that renal function decline was much more marked with partial nephrectomy; it was virtually negligible with RFA.

There are substantial advantages in using thermal ablation to treat small renal neoplasms: in most patients the procedure can be carried out with intravenous analgesia, local anesthesia and sedation, with a short stay in hospital. Renal function is affected much less than with partial nephrectomy. There is reduced overall morbidity, and thermal ablation is certainly less expensive than surgery.
We did a small pilot study on quality of life, comparing robotic partial nephrectomy with ablation and found temporary reduction in quality of life with robotic partial nephrectomy but not with ablation. We have also compare costs; of course, these will vary from hospital to hospital and health-care system to health-care system, but at guy's and St. Thomas's, robotic partial nephrectomy actually cost us a 112 pounds because we are reimbursed less than we receive for the operation, whereas RFA leaves us in the black by three and half thousand pounds.

The advantages of RFA for the treatment of small renal tumors are now recognized in international guidelines. The American Society for Clinical Oncology guidelines used to say that thermal ablation should be used when surgery is not possible. They no longer say that; the only condition they put on the use of thermal ablation as first-line treatment is that complete ablation should be achievable. You might think that people who work in this area like me who would be very pleased with this, and I am. However, I believe that the guidelines should go further, and that tumors that are ideal for ablation-those that are smaller than $3 \mathrm{~cm}$, posterior, exophytic and away from the pelviureteric junction-should be treated with thermal ablation in preference to surgery. Many urologists would argue that before one can make such a statement a randomized study would need to be carried out. However, we have calculated that in order to reach $90 \%$ power for such a comparison, the study would need to recruit would 10,800 patients, which is unlikely to happen.

This brings me to a few points that I would like to make in relation to the nature of evidence in medicine. In 2008, Professor Sir Michael Rawlins, who was the first chairman of the National Institute for Clinical Excellence in the United Kingdom, gave the Harveian Oration, which is most prestigious medical lecture in Britain. He called it De Testimonio, because it dealt with the nature of evidence in medicine and argued that the notion of hierarchies of evidence is illusory. He also quoted Bradford Hill, who is the architect of the randomized controlled trial. Bradford Hill said that "any belief that the controlled trial is the only way would mean not that the pendulum had swung too far but that it had come right off the hook." Of course, Michael Rawlins is not against randomized controlled trials (RCTs). He is a distinguished pharmacologist and he considers RCTs the best way to investigate certain issues in medicine. The point his making here is that they are not always the best way to analyze certain types of evidence.

This issue can be particularly problematic when analyzing the results of procedures and surgical operations, because it is not always possible to randomize for the skill of the operator. Also changing technology can invalidate the study before it is finished. In the potential study of I mentioned, concerning robotic partial nephrectomy versus RFA, where you need almost 11,000 patients, advances in 
technology could invalidate the study before it could be completed. When we cannot carry out randomized trials we can do large prospective studies, registries, and look at patient-reported outcomes.

Now let me come to some points regarding research. There is a lot of work on the combination of ablation and immunotherapy. This is a very exciting field. Ablation kills cancer cells, which act-like antigens. They come into contact with immune cells and cytokines, thus priming the immune system and promoting immunogenic cell death. There is some evidence that there is synergy between RFA and immunotherapy. For example in a small study on patients with hepatocellular carcinoma, in which RFA was compared with RFA plus immunotherapy, the combined treatment resulted in a longer progression-free survival.

I am grateful to Dr. Thanou, from King's College London, for lending me these images, which illustrate her research with nanoparticles. She is using doxorubicin-containing liposomes, labeled with gadolinium, which can be imaged with magnetic resonance imaging. As they pass through liver tumors they can be treated with high intensity focused ultrasound, which heats the lipid envelope and bursts it, thus releasing the doxorubicin in high concentration within the tumor, whereas it remains in low concentration systemically. Nanoparticles may have application in thermal ablation. There is a high edge-recurrence rate when liver tumors are treated with thermal ablation because cells at the periphery of the treated area may not get hot enough to be killed. It may be possible to inject doxorubicin-containing nanoparticles during thermal ablation. The heat generated by the ablation at the edge of the treated area may not be sufficient to kill the malignant cells by itself, but by bursting the nanoparticles it may release doxorubicin in sufficiently high concentration to reduce the rate of local recurrence.

Quality assurance is an integral part of clinical work in disciplines such as radiation oncology, but has been virtually ignored until now in in interventional oncology. It is time to change this situation, because it can lead to many deficiencies. For example, many interventional radiologists engaged in cancer care do not follow their patients personally for long enough to detect residual or recurrent tumor and delegate this task to the referring oncologist. However, this can lead to confusion and delay, and as a result some treatable residual tumors can become untreatable. Another example concerns the training of radiographers: it would unthinkable for radiographers delivering radiotherapy not to be specifically trained for what they do; but the majority of interventional oncologists is assisted during ablations by radiographers with limited or incomplete training in this field.

Recognizing this deficiency, CIRSE set about developing standards for quality assurance in interventional oncology. These concern the whole of the patient pathway. The aim is to increase patient safety and encourage good practice.
There are three sections in the standards that CIRSE has developed: staff and facilities, treatment planning and delivery, and safety and quality. Under each section there are is a set of standards. Some of the standards are technical like "Medical devices and equipment." Others are more clinical, such as "Patient care during interventional treatment delivery." The standard is a goal or an outcome. Under each standard there are criteria that describe how this is to be done and then there is a detailed commentary under each criterion, which provides much more detail about how this should take place in everyday practice. And then, there is the required evidence: a list of documents and records that a facility engaging in the interventional oncology would need to produce to demonstrate compliance with the standards. For example under "facility process management" there is standard that talks about interventional oncology care being deliver in the timely fashion and in a coordinated way. The criterion describes coordination of the patient and referral pathways. The commentary goes into greater detail. For example, it mentions participation by interventional oncologists in multi-disciplinary team meetings.

The standards have now been endorsed by 25 national and international radiological societies around the world and they serve as the basis for a credentialing system called International Accreditation System for Interventional Oncology Services (IASIOS), which stands for IASIOS. IASIOS promotes excellence and a high quality of patient care. Furthermore, it provides an opportunity for facility to demonstrate its achievements.

IASIOS is an interactive process. When a facility applies for the accreditation under this system, a dialog begins between the facility itself and a dedicated team in a CIRSE office, the IASIOS team. A preliminary look takes place and then advice is offered to the facility on how it could improve its performance in certain areas and when the facility considers that it is close to reaching the required level of practice an informal submission is made and a proper look takes place. At that point, more advice is offered. When the facility thinks it is ready and the team agrees, a formal submission is made. At that point, the IASIOS committee, which consists of several eminent interventional oncologists, formally considers the evidence. If the standards have been met, accreditation is granted and the facility becomes an IASIOS accredited center. If they have not been met, more advice is offered and more work is done before another submission is made.

Some of the standards are core, and have to be met by any facility that applies for accreditation. Others are more ambitious and more aspirational, and any facility that meets even those would become an IASIOS center of excellence. I believe that this accreditation system will help interventional oncology to become a proper clinical discipline. 
We started a formal pilot phase in the summer of 2018, involving six centers-three in Europe, two in Australia, and one in Asia. More are about to join now. The results of the pilot will help us to refine IASIOS that further refinement of IASIOS before a formal public launch in 2021.

Interventional oncology has the potential to make a huge contribution to modern cancer care. I believe that 10 years from now most local tumor treatment will be delivered either with sophisticated methods of radiotherapy or by interventional oncological techniques. Surgery will be largely reserved for large tumors in solid organs and for tumors in hollow organs. I am convinced that, by then, interventional oncology truly will be a mainstream part of modern cancer care.

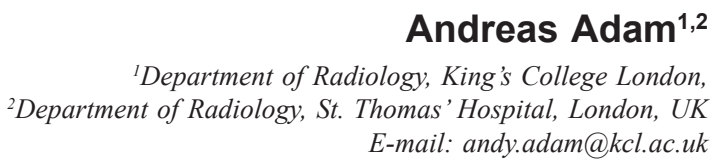

Andreas Adam ${ }^{1,2}$

${ }^{\prime}$ Department of Radiology, King's College London, ${ }^{2}$ Department of Radiology, St. Thomas' Hospital, London, UK E-mail:andy.adam@kcl.ac.uk

This is an open access journal, and articles are distributed under the terms of the Creative Commons Attribution-NonCommercial-ShareAlike 4.0 License, which allows others to remix, tweak, and build upon the work non-commercially, as long as appropriate credit is given and the new creations are licensed under the identical terms.

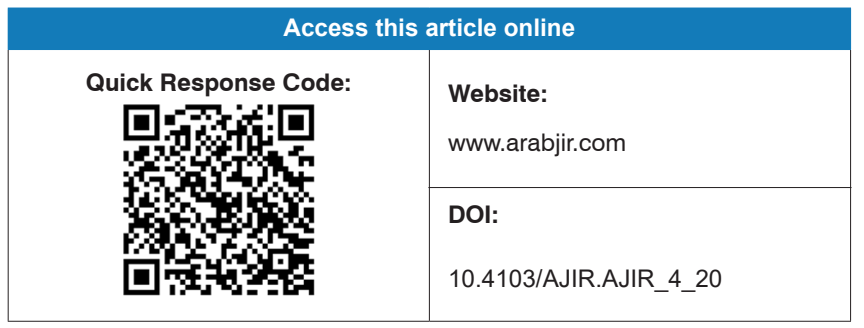

How to cite this article: Adam A. Interventional oncology: A key part of mainstream cancer care. Arab J Intervent Radiol 2020;4:53-6.

Received: 24-03-2019

Accepted: $24-03-2020$

Online Published: 25-04-2020 